\title{
Ultrasonic Array Imaging through Reverberating Layers for Industrial Process Analysis
}

\author{
${ }^{1 *}$ Marcus Ingram, ${ }^{1}$ Anthony Gachagan, ${ }^{2}$ Anthony J. Mulholland, ${ }^{3}$ Alison Nordon and ${ }^{4}$ Martin Hegarty \\ ${ }^{1}$ Centre for Ultrasonic Engineering, ${ }^{2}$ Dept. Mathematics and Statistics, ${ }^{3}$ Dept. Pure and Applied Chemistry \\ ${ }^{1-3}$ University of Strathclyde, Glasgow, UK \\ ${ }^{4}$ BP Chemicals Limted, Hull, UK \\ *m.ingram@strath.ac.uk
}

\begin{abstract}
In this paper, ultrasonic phased arrays are investigated as an imaging tool for industrial process analysis. Noninvasive process measurement, via transmission of information through a vessel wall, typically requires a window to create an optical path between the sensor and the process. Ultrasonic array imaging provides a means to overcome this barrier as it is specifically used to image into optically opaque structures. However, the large acoustic impedance mismatch between the steel process vessel and water load results in reverberations clouding the image scene containing reflections from within the process fluid. A methodology to identify and remove this reverberation interference from the image scene is proposed using subspace analysis coupled with phase coherence imaging. A 32 element, $5 \mathrm{MHz}$ finite element array model mounted to the outside of a steel vessel wall is used to demonstrate the application of this methodology to a typical industrial process environment. The final image is free of reverberation artefacts, providing a means to accurately extract quantitative information about the process from these images.
\end{abstract}

Index Terms - ultrasonic phased array, industrial process analysis, non-invasive process imaging

\section{INTRODUCTION}

Industrial process analysis is the measurement of information used to control an industrial process. Correct control of an industrial process increases workplace safety, improves consistency of product quality and improves the environmental impact by reducing waste [1]. Under ideal conditions, the measurement sensor is positioned non-invasively without direct contact with the process. This reduces measurement uncertainty from disturbances to the flow and reduces the measurement cost as the hardware can be easily replaced without interruption to the process [2].

Ultrasound is typically deployed to non-invasively image into optically opaque structures, such as steel [3]. Therefore, ultrasound imaging provides a well-established technological solution to achieve non-invasive visualisation of industrial processes. In this paper, a methodology has been proposed to generate images of reflectors positioned within a fluid process via a steel layer. These images can then be post-processed to extract quantitative information about the process.

At the interface between the steel vessel wall and the process fluid (water), there is a large acoustic impedance

Engineering and Physical Sciences Research Council (grant number EPM5076471) mismatch. This creates a strongly reflecting acoustic boundary where approximately $87.5 \%$ of the ultrasonic energy injected into this system is reflected. Therefore, signals reflected from within the process are inherently weak relative to signals reflected from the steel-fluid acoustic boundary. In addition, the ultrasonic energy reflected at this acoustic boundary reverberates within the steel vessel wall, masking signals reflected from within the process.

Non-invasive ultrasonic images of industrial processes can be generated using ultrasonic tomography. The data acquisition rate is low relative to pulse-echo phased arrays because the ultrasonic path length must cross the entire diameter of the pipe [4]. Therefore these images do not contain detailed quantitative information about the process. Also, Perspex vessel material has been typically used to aid ultrasonic transmission into the process and this requires modification of the vessel, reducing the measurement flexibility [5].

The aim of this work was to develop a non-invasive throughsteel ultrasonic imaging system to extract detailed quantitative information about an industrial process. The target application was imaging of bubbles rising to the surface of a fluid in a reactor or flow through a pipe. A schematic of the ultrasonic hardware coupled to the target application is presented in Figure 1. For clarity in the results presented in this paper, the steel vessel wall was not included in the image scene and the $\mathrm{z}$-axis of the image begins at the steel-fluid interface.

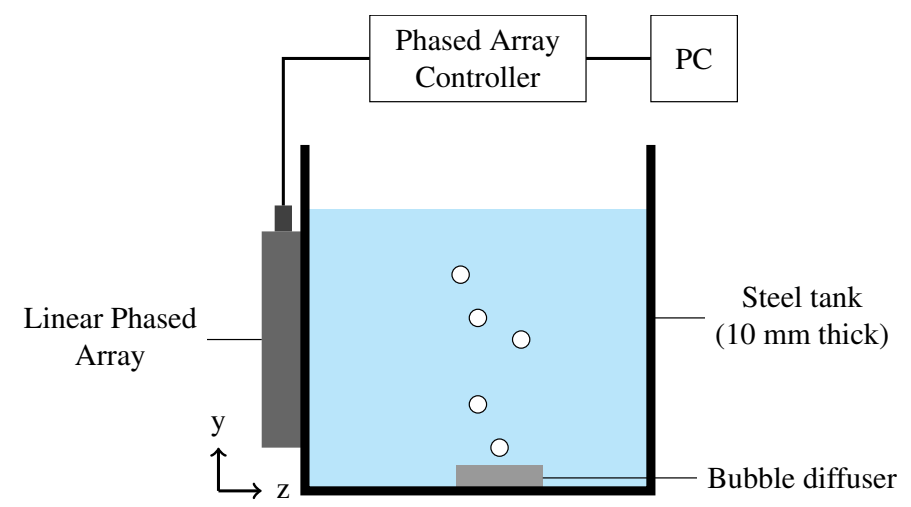

Fig. 1. Schematic of the ultrasonic hardware coupled to image target. 


\section{Methodology}

\section{A. Data Acquisition and Imaging}

To maximise the quantity of information acquired about the image scene, an ultrasonic phased array was deployed using Full Matrix Capture (FMC) [6]. The image scene, constructed using the Total Focusing Method (TFM) [6], had $i$ pixels in the $y$ dimension and $j$ pixels in the $z$ dimension. Using the delay and sum approach for $M$ A-Scan time domain signals, $y_{m}(t)$, the intensity at a given pixel, $I_{i j}$, was given by

$$
I_{i j}=\sum_{m=1}^{M} y_{m}\left(\tau_{i j}-t_{0}\right),
$$

where $\tau_{i j}$ was the time-of-flight between the transmitting element $\left(T_{x}\right)$, the pixel and the receiving element $\left(R_{x}\right)$ and $t_{0}$ was the start time. A drawback to the TFM is signal aliasing; signals from two different points in space can incorrectly combine, contributing to the value of a pixel where no real reflector exists. This means the image scene in this work was not temporally resolved because the reverberations in the steel were mapped onto the pixels corresponding to the process fluid. Note that refraction at the steel-fluid acoustic interface was incorporated into the calculation of $\tau_{i j}$ using a dual-layer TFM algorithm [7].

\section{B. Finite Element Model}

A 32 element, finite element model was designed in PZFlex (OnScale, USA) to generate an FMC data set of the target image scene, shown in Figure 2. The transducer was modelled as a pressure loaded array, meaning no transducer effects were modelled and it was assumed the device was perfectly coupled to the outside wall of the steel vessel. This simplified design provided ground truth that any variation between results were purely due to the integrity of the methodology rather than noise from the array design.

The driving function selected was a $5 \mathrm{MHz}$ BlackmanHarris function to reduce any side-lobe activity. The thickness

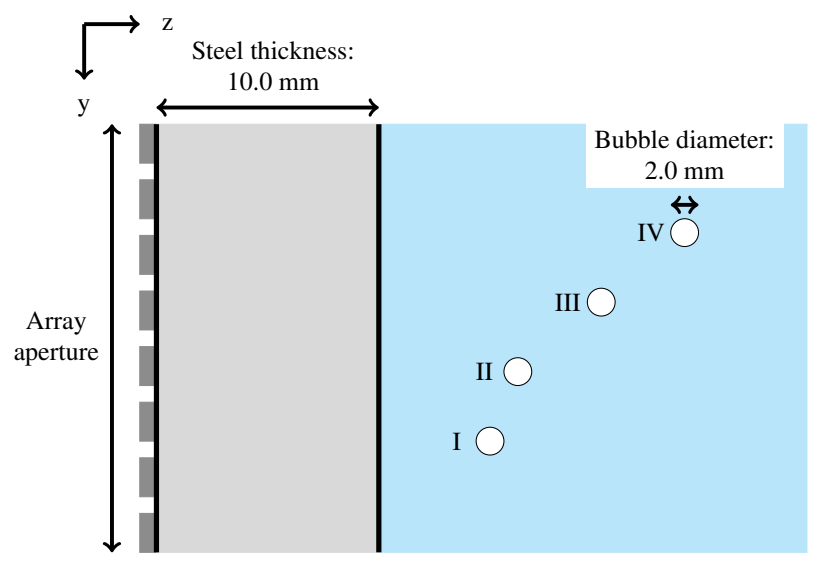

Fig. 2. Schematic of PZFlex model used to generate a non-invasive FMC data set. of the steel vessel wall was set to $10 \mathrm{~mm}$ and the bubble reflectors were modelled as $2 \mathrm{~mm}$ diameter circular voids suspended in water. The position of the voids was designed so that signal aliasing occurred between the reverberation signals and voids I and III but not voids II and IV. The boundary conditions were set to absorbing to create a system with adequate reverberations but without artefacts from the transducer or elsewhere. A background FMC data set was generated by repeating the model without the voids present. The background subtracted response provided a benchmark for the reverberation removal method proposed but in practice this data set would not be available in a process environment.

\section{Reverberation Removal Methodology}

The proposed methodology to remove reverberation artefacts from the image scene was to combine subspace analysis [8] with Phase Coherence Imaging (PCI) [9]. Relative to the regular TFM imaging process, the procedure does not rely on any additional information about the image scene to identify and remove the reverberation artefacts. This prevented measurement uncertainty impacting on the outcome of the reverberation removal procedure because it was purely dependent on the raw FMC data.

Consider an FMC data set, $Y$, acquired using a $K$ element aperture with dimensions $M=K^{2}$ A-Scans by $N$ time samples. If $Y \in \mathbb{R}^{\mathrm{M} \times \mathrm{N}}$ arose from an array mounted to the outside wall of a steel vessel, there will be wall reverberations intrinsic to all the measured data. These reverberations, $r(t)$, are in addition to the signals of interest, $s_{f}(t)$, from within the process and measurement noise, $n(t)$. Therefore, each A-Scan, $y_{m}(t)$, can be represented by three terms

$$
y_{m}(t)=s_{f}(t)+r(t)+n(t) .
$$

This assumes that both the signals of interest and noise are completely independent of the reverberation signals. The spatial orientation of the void reflectors is not consistent for all element locations in the aperture, however, the spatial position of the reverberating interface is consistent for any $T_{x}-R_{x}$ pair in the aperture data. Therefore, the reverberation signals in any A-Scan in the aperture data must have a high degree of coherency relative to the signals from the void reflectors. The subspace analysis methodology proposes that this can be exploited to specifically extract the reverberation signals from the FMC data set.

The covariance matrix, $C_{Y} \in \mathbb{R}^{\mathrm{M} \times \mathrm{M}}$, of the FMC data is

$$
C_{Y}=\frac{1}{M} Y Y^{T}
$$

where $[\cdot]^{T}$ denotes the transpose operator. The covariance matrix is then decomposed into its latent variables using Eigen decomposition,

$$
C_{Y}=\sum_{m=1}^{M} \lambda_{m} \mathbf{u}_{m} \mathbf{u}_{m}^{T},
$$

where $\lambda_{m}$ is the $m^{t h}$ eigenvalue ordered from largest to smallest and $\mathbf{u}_{m}$ represents its corresponding eigenvector. The 
first $K$ eigenvectors describe the variation in $Y$ resulting from the signals of interest, whereas the remaining $\eta=M-K$ eigenvectors describe the variation in $Y$ resulting from the reverberations and noise. This is because the spatial positions of the reflectors of interest vary relative to the element that is transmitting, whereas the spatial positions of the reverberating interface and noise do not change, independent of which element is transmitting.

From the last $\eta$ eigenvectors in the ordered set the reverberation subspace, $U_{r} \in \mathbb{R}^{\mathrm{M} \times \eta}$, was constructed

$$
U_{r}=\left[\begin{array}{llll}
\mathbf{u}_{K+1} & \mathbf{u}_{K+2} & \ldots & \mathbf{u}_{M}
\end{array}\right] .
$$

When $U_{r}$ was projected onto the FMC data, the signals corresponding to the reverberation clutter and noise were weighted relative to the signals of interest, which had the impact of removing them from the FMC data,

$$
\hat{s}_{f}=\left(U_{r} U_{r}^{T}\right) Y,
$$

where $\hat{s}_{f} \in \mathbb{R}^{\mathrm{M} \times \mathrm{N}}$ was the modified FMC data used to construct the image scene.

The second stage was to perform PCI with this modified FMC data set in parallel with TFM image construction. The PCI and TFM processes can be implemented in real-time because there is an intrinsic overlap in how these two processes operate.

The regular TFM process maps the amplitude of aperture data at each pixel in the image scene, whereas PCI maps the phase distribution of the aperture data at each pixel location. The phase distribution of the aperture data is termed the Phase Coherence Factor (PCF), which is used to weight the pixel values in the final TFM image. This is possible because the standard deviation of the phase from a perfectly focussed scatterer will always be zero, whereas phase artefacts from side lobe activity deviate the phase distribution towards one. Therefore the PCF becomes a matrix with the same dimensions as the TFM image, although with values ranging from 0 to 1 for each pixel corresponding to the coherence of the focal point with the main beam.

The instantaneous phase of the aperture data, $\psi(t)$, can be determined from the real and imaginary terms of the Hilbert Transform of the FMC data,

$$
H(t)=S_{r}(t)+j S_{i}(t),
$$

where $S_{r}(t)$ and $S_{i}(t)$ represent the in-phase and quadrature components of the aperture data. From this analytical signal representation, the instantaneous phase of the FMC data is calculated

$$
\psi(t)=\tan ^{-1}\left(\frac{S_{i}(t)}{S_{r}(t)}\right) .
$$

For a given pixel, $\psi(t)$ is calculated $M$ times and the value of the PCF at that pixel location is calculated from

$$
P C F_{i j}=\max \left[0,1-\frac{\sigma(\psi)}{\sigma_{0}}\right]
$$

where $\sigma(\psi)$ is the standard deviation of the instantaneous phases of the FMC data and $\sigma_{0}=\pi / \sqrt{3}$ represents the standard deviation of a uniformly distributed random variable.

\section{RESULTS AND DISCUSSION}

The regular TFM process was applied to the simulated FMC data set with the four void reflectors. The resulting image was recorded in Figure 3, where the black circles represent the true location of the voids. Figure 3 illustrates the impact of the reverberations on the signal-to-noise ratio (SNR) of the void reflectors, where the high-amplitude reverberations set the dynamic range of the image, masking the void reflectors.

The regular TFM process was applied to the background subtracted FMC data set, shown in Figure 4. The background subtracted image revealed the locations of the void reflectors by removing artefacts corresponding to the main-beam reverberations. However, regions of the image scene beyond the voids exhibited a lower SNR because ultrasound reflected from the voids reverberated within the steel creating further artefacts that were mapped onto the image scene. These void reverberations were less coherent across the array aperture than the main-beam reverberations so could not be removed using subspace analysis. It was for this reason it was proposed to combine subspace analysis with PCI.

Upon Eigen decomposition of the FMC covariance matrix, the explained variance captured in the first $K$ eigenvectors was $99.994 \%$. This highlights the variation described by the signals of interest relative to the reverberations and noise.

The reverberation and noise subspace was projected onto the original FMC data using Equation 6 and these modified FMC data were constructed into a TFM image that was weighted by the PCF. The image resulting from this process was recorded in Figure 5. In this image the void reflectors were revealed

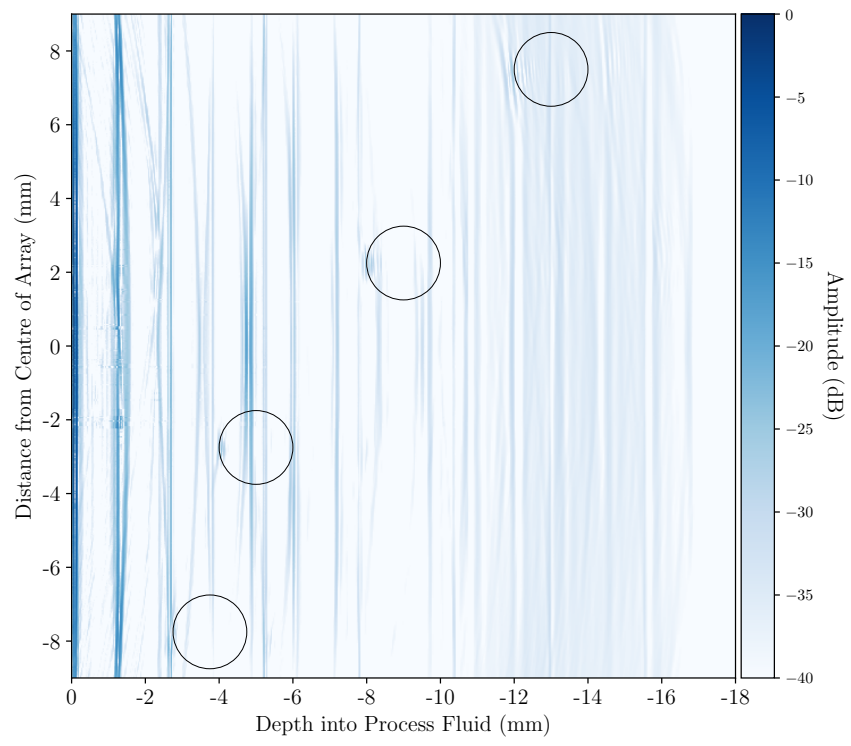

Fig. 3. TFM image from simulated FMC data without any reverberation removal prior to image construction. 


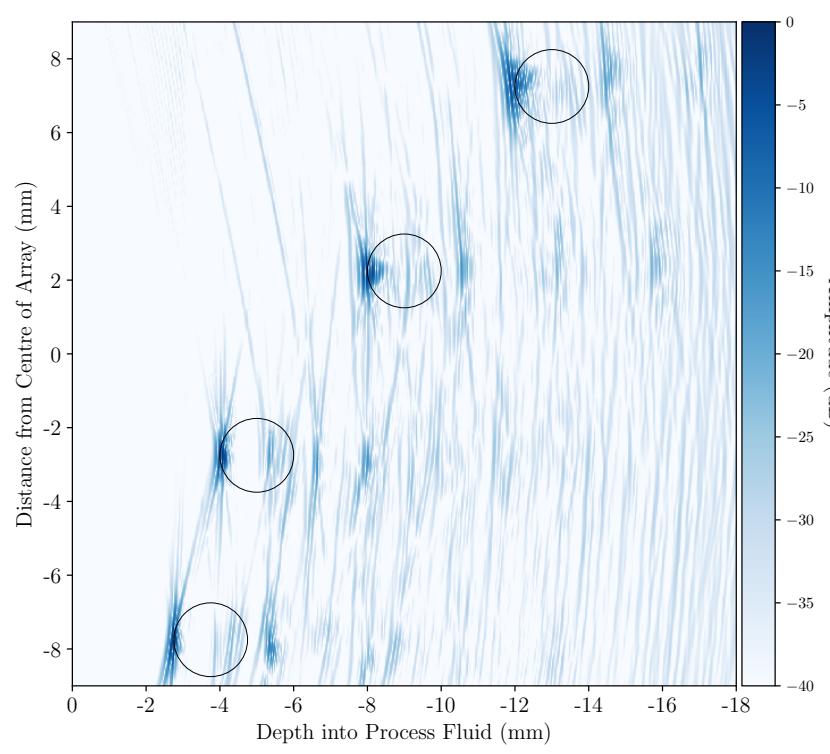

Fig. 4. Result from background subtraction of simulated FMC data prior to TFM image construction.

by the application of subspace analysis and artefacts corresponding to the void reverberations were suppressed by the application of PCI. Given that voids I and III were positioned to aliase with the main-beam reverberations, this highlights the robustness of this methodology to separate signals relating to the reverberations and noise from the signals of interest.

The application of subspace analysis followed by PCI has resulted in an image free from reverberation clutter. This enables quantitative information relating to the position and size of these reflectors to be accurately extracted from these

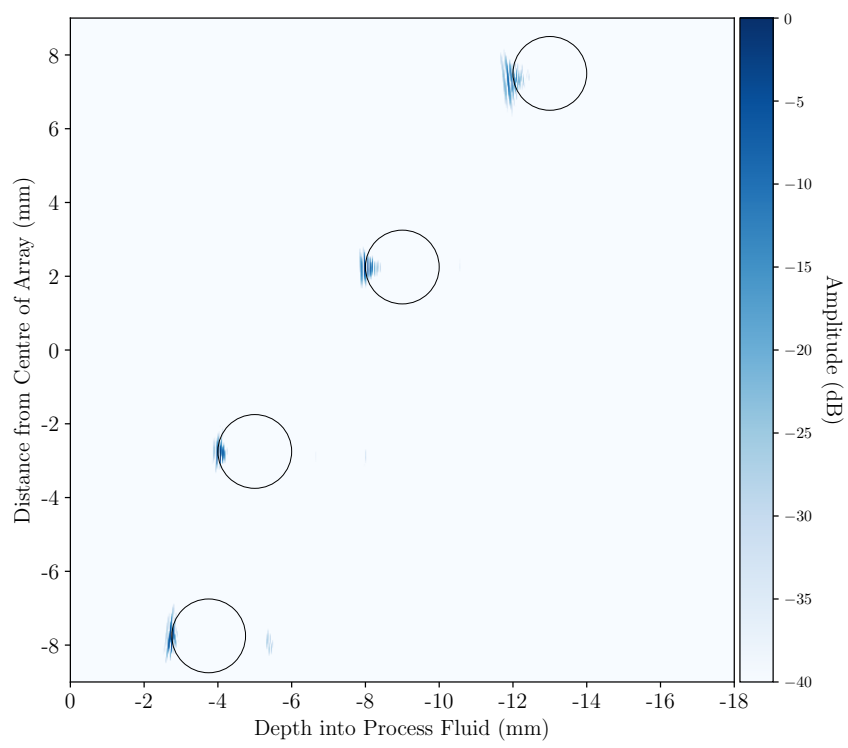

Fig. 5. Image scene of void reflectors after application of subspace analysis and PCF applied to the TFM image construction. non-invasive process images. For example, this could be used to extract the bubble size distribution of a dynamic process.

\section{CONCLUSIONS}

Non-invasive through-steel ultrasonic imaging of a typical process environment has been achieved using a combination of subspace analysis and PCI. The proposed methodology is standalone as it does not require any additional information about the imaging system, other than the raw aperture data, to be implemented. The findings were verified using a simulated FMC data set, which showed complete removal of artefacts corresponding to reverberations from the main-beam and from reflectors within the process fluid. The result creates the opportunity to develop a non-invasive through-steel imaging system for industrial process analysis.

\section{ACKNOWLEDGMENT}

The authors would like to thank BP Chemicals Limited for their support and industrial direction.

\section{REFERENCES}

[1] J. B. Callis, D. L. Illman, and B. R. Kowalski, "Process Analytical Chemistry," Analytical Chemistry, vol. 59, no. 9, pp. 624-637, 1987.

[2] F. McLennan, "Process Analytical Chemistry in Perspective," in Process Analytical Chemistry. Springer, 1995, pp. 1-13.

[3] B. Drinkwater and P. Wilcox, "Ultrasonic Arrays for Non-Destructive Evaluation: A Review," NDT \& E International, vol. 39, no. 7, pp. 525 $-541,2006$.

[4] B. Hoyle, "Process Tomography using Ultrasonic Sensors," Measurement Science and Technology, vol. 7, no. 3, pp. 272 - 280, 1996.

[5] L. Xu and L. Xu, "Gas-Liquid Two-Phase Flow Regime Identification by Ultrasonic Tomography," Flow Measurement and Instrumentation, vol. 8, no. 3, pp. $145-155,1997$.

[6] C. Holmes, B. Drinkwater, and P. Wilcox, "Post-Processing of the Full Matrix of Ultrasonic Transmit-Receive Array Data for Non-Destructive Evaluation," NDT \& E International, vol. 38, no. 8, pp. 701 - 711, 2005.

[7] J. Dziewierz, T. Lardner, and A. Gachagan, "A Design Methodology for 2D Sparse NDE Arrays using an Efficient Implementation of RefractedRay TFM," in 2013 IEEE International Ultrasonics Symposium (IUS). IEEE, 2013, pp. 136-138.

[8] R. Demirli, M. G. Amin, X. Shen, and Y. D. Zhang, "Ultrasonic Flaw Detection and Imaging Through Reverberant Layers via Subspace Analysis and Projection," Advances in Acoustics and Vibration, vol. 2012, pp. 1-10, 2012.

[9] J. Camacho, M. Parrilla, and C. Fritsch, "Phase Coherence Imaging," IEEE TUFFC, vol. 56, no. 5, pp. 958 - 974, 2009. 